\title{
Clinicopathological Features and Prognosis of Pregnancy Associated Breast Cancer - A Matched Case Control Study
}

\author{
Lilla Madaras • Kristóf Attila Kovács • Attila Marcell Szász • \\ István Kenessey • Anna-Mária Tőkés • Borbála Székely • \\ Zsuzsanna Baranyák • Orsolya Kiss • Magdolna Dank • Janina Kulka
}

Received: 23 August 2013 / Accepted: 5 December 2013

(C) Arányi Lajos Foundation 2013

\begin{abstract}
Pregnancy Associated Breast Cancer (PABC) manifests during pregnancy or within a year following delivery. We sought to investigate differences in management, outcome, clinical, histopathology and immunohistochemistry (IHC) characteristics of PABC and matched controls in a retrospective case control study. $\mathrm{PABC}$ and control patients were selected from breast cancer cases of women $\leq 45$ years, diagnosed in the 2nd Department of Pathology, Semmelweis University, Budapest, Hungary between 1998 and 2012. Histopathology information on tumor type, grade, size, T, N, lympho-vascular invasion (LVI), Nottingham Prognostic Index (NPI), associated in situ lesions and IHC charcteristics: ER, PgR, HER2, Ki67, p53 were recorded, IHC-based subtype was assessed, clinical, management and outcome data were analysed. Thirty-one breast cancer cases were pregnancy related. Clinical management data did not differ in cases and controls. Histopathology of disease at presentation was not significantly different, but NPI assessed the PABC group as
\end{abstract}

Electronic supplementary material The online version of this article (doi:10.1007/s12253-013-9735-9) contains supplementary material, which is available to authorized users.

L. Madaras $(\bowtie) \cdot$ K. A. Kovács • A. M. Szász • I. Kenessey •

A.-M. Tőkés · B. Székely • Z. Baranyák • O. Kiss · J. Kulka

2nd Department of Pathology, Semmelweis University, Üllöi út 93., 1091 Budapest, Hungary

e-mail: 1illa.madaras@gmail.com

Z. Baranyák

Department of Surgery, Medical Center, Hungarian Defense Forces,

Róbert Károly krt. 44, 1134 Budapest, Hungary

M. Dank

Division of Oncology, 1st Department of Internal Medicine, Semmelweis University, Tömő u 25-29, 1083 Budapest, Hungary

A.-M. Tőkés

Hungarian Academy of Sciences, MTA-SE Tumour Progression

Research Group, Budapest, Hungary having poor, whereas controls as having intermediate prognosis. Associated in situ lesion was more often high grade Extensive Intraductal Carcinoma Component (EIC) in PABC. Triple negative and LuminalB prol tumors predominated in PABC. Disease-free and overall survival was inferior compared to controls. PABC patients with LuminalB prol and Triple negative tumors had inferior outcomes. On multivariate analysis inferior prognosis of PABC was associated with pregnancy. Our study has demonstrated inferior outcome of PABC. Difference in tumor biology is reflected by the predominance of triple negative and LuminalB tumors in PABC. The strength of the study is the analysis of complete pathology and IHC data.

Keywords Breast cancer · Pregnancy associated breast cancer $\cdot$ Pregnancy $\cdot$ Postpartum $\cdot$ Young women

\section{Introduction}

Pregnancy associated breast cancer (PABC) has been most commonly defined as breast cancer complicating pregnancy, manifesting during lactation or within 1 year after delivery. The definition is not unanimous however, some authors define the postpartum time frame from 6 months to 2 years after delivery $[1,2]$.

Pregnancy has dual influence on breast cancer risk, it has long-term protective effect but epidemiologic data also demonstrate a transient increase in breast cancer incidence postpartum. The postpartum time frame for this increased risk ranges from 2 to 15 years, or even longer in case of older first time pregnant women [3-5].

The incidence of PABC is estimated to be $1 / 10000$ to $1 / 3000$ pregnancies in Western countries, which is expected to rise due to the trend of women postponing childbearing to 
later age. It is estimated that $10 \%$ of breast cancer cases affecting women $\leq 40$ years of age are pregnancy related [6].

The situation is complicated at social, ethical, psychological and medical levels, since the diagnosis is often challenging due to physiologic changes occuring in the breast during pregnancy or lactation, and because treatment of the pregnant mother-however essential — can potentially harm the fetus. Clinicians have very limited experience in this complex setting, and this may lead to delayed diagnosis, delayed or undertreatment of maternal breast cancer, controversial termination of pregnancy or induced premature delivery.

The prognosis of PABC is more often reported as being unfavorable $[1,7-10]$. Whether this poor prognosis is related to pregnancy or mainly due to the young age of patientswhich in itself is known to be a poor prognostic factor [11] - is not clear.

Studies on PABC are rare, with only a few addressing the pathological characteristics of the disease. The majority supply information on the invasive tumor's type, grade and TNM stage, together with only the immunohistochemistry (IHC) assessment of hormonal receptors (with incomplete data provided by most of the studies); data on Her2 status [12-19], Ki67 labeling index [12, 15, 17], p53 $[12,18]$, IHC-based tumor subtype [15] as well as on the characteristics of eventual associated in situ lesions [19] are scarce. To the best of our knowledge this is the first matched case control study that combines information on the management and outcome of PABC patients together with analysis of all the relevant histopathology and IHC information the pathologist can provide on the multidisciplinary approach to PABC.

The aim of this retrospective matched case control study was to investigate differences in clinicopathological features, immunophenotype, treatment and outcome of PABC and matched control, non-PABC cases.

\section{Patients and Methods}

First, upon specific approval of the Semmelweis University Institutional Review Board (TUKEB 17/2006, 3/2013) we reviewed the database of the 2nd Department of Pathology, Semmelweis University and recorded all the breast cancer cases of women $\leq 45$ years of age, diagnosed between January 1st, 1998 and November 1st, 2012. Histopathological data of the invasive tumor were obtained: tumor type, Nottingham grade, multifocality, characteristics of eventual associated in situ lesions, invasive tumor size, regional lymph node involvement, TNM stage, Nottingham Prognostic Index (NPI), presence of lympho-vascular invasion (LVI), and the characteristics of IHC analysis were investigated: Estrogen receptor (ER), Progesterone receptor (PgR) and Her2 expression, Ki67 labeling index and p53 expression. Used antibodies, methodology and FISH assessment method are summarized in Supplementary Material 1. ER and PgR statuses were evaluated according to the most recent guidelines [20], Ki67 positivity was measured as the ratio of positive tumor cell nuclei in the tumor and Her2 IHC was evaluated according to current guidelines [21] by a $0-3$ scale standard protocol. FISH results were evaluated according to standard protocol [21]: non-amplified if the HER2/CE17 ratio was less than 1.8, equivocal if this ratio was between 1.8 and 2.2 and amplified if the HER2/CE17 ratio was over 2.2. When discordance occurred with Her2 IHC, FISH results were taken into consideration.

A tumor was considered ER and/or PgR positive if $10 \%$ of tumor cells demonstrated intranuclear positivity. In Ki67 IHC assessment, a labeling index of $14 \%$ or more was considered high [22]. Based on IHC characteristics of the invasive tumor, the IHC-based subtype was assessed. ER (and eventually $\mathrm{PgR}$ ) positive tumors with low Ki67 labeling index were considered Luminal A (LumA), ER (and eventually PgR) positive tumors with high Ki67 labeling index or HER2 expression were considered Luminal B subtype (LumBprol or LumBHer2 respectively). The tumors which were hormone receptor (HR) negative and expressed Her2 were considered as Her2 positive, while tumors that did not express either HRs or Her2 were considered as triple negative (TNBC). [23]

In the second step, we reviewed the patient database of Semmelweis University for recorded breast cancer patients and added all the available loco-regional and systemic treatment information, relevant personal, parity and family history, as well as data on eventual loco-regional and systemic relapses. Finally, we selected all pregnancy associated breast cancer cases manifesting during pregnancy, lactation or within 1 year after delivery.

Control patients were identified through the two databases, each PABC patient was matched by age $( \pm 1$ year) and year of first breast cancer (BC) diagnosis ( \pm 1 year) to a control patient with non-PABC.

Asymmetrical numeric data were analyzed by matched Wilcoxon-test. Categorical data were compared using Chisquare and Fisher's exact test. Overall survival analyses were performed using the Kaplan-Meier method. Overall survival intervals were determined as the time period from initial diagnosis to the time of death or date of last follow up. Disease-free survival was calculated as the time interval from initial breast cancer diagnosis to the date of disease recurrence (loco-regional or systemic) or to time of death from BC, or to the date of last follow-up if the patient was disease free. Comparison between survival functions for different strata was assessed with the log-rank statistic. Multivariate analysis of prognostic factors was performed using Cox's regression model. Differences were considered significant when $p \leq 0.05$. All statistical analyses were performed using Statistica 9.0 software (StatSoft Inc. Tulsa, OK). 


\section{Results}

\section{Clinical Data Analysis}

Thirty-one breast cancer cases were found to be pregnancy related, 10 manifesting during pregnancy and 21 during lactation or postpartum period, within 1 year after delivery. Patient characteristics and management data of all PABC and control patients are summarized in Table 1. Median age at diagnosis was 34 (range: 29-42) years for both the PABC and control group (range: 28-42).

\section{Treatment Modalities During Pregnancy}

Among the pregnant patients three were diagnosed with breast cancer during the first trimester, six during the second trimester and one in the third trimester of their pregnancy.

Six patients underwent surgery (four breast conserving surgery/BCS/and two mastectomy, all with axillary lymph node dissection/ALND/) while pregnant, followed by adjuvant therapy after delivery in four cases. One patient received neoadjuvant chemotherapy (2xFAC) followed by surgery (mastectomy with
ALND) during pregnancy and adjuvant therapy after delivery. One patient had surgery (BCS with ALND) followed by adjuvant chemotherapy $(6 \mathrm{xFEC})$ while pregnant.

\section{Pregnancy Outcome}

One patient underwent induced abortion at 23rd week of gestation, five patients had elective cesarean section (between the 29th and 34th week of gestation) and four patients delivered their babies spontaneously (one preterm delivery).

Treatment Modality Comparison of PABC and Control Cases

There was no significant difference in the modality of surgery (breast conserving surgery versus mastectomy) between cases and controls. Axillary lymph node dissection was the main therapy of choice in both groups. The pregnancy associated group did not show significant difference in elapsed time between diagnosis and surgery compared to controls $(p=0.4$, data not shown). Neoadjuvant, adjuvant chemo- and radiotherapy frequency or regimen did not differ $(p=0.839)$ between the cases and controls. Adjuvant endocrine therapy was

Table 1 Patients characteristics and management data of PABC and control patients

\begin{tabular}{|c|c|c|c|c|c|}
\hline Age & & median (years) & $\begin{array}{l}\mathrm{PABC} N=31 \\
34\end{array}$ & $\begin{array}{l}\text { Control } N=31 \\
34\end{array}$ & $\begin{array}{l}p \text {-value } \\
\text { match }\end{array}$ \\
\hline \multirow[t]{5}{*}{ Surgery } & \multirow[t]{3}{*}{ Breast } & $\begin{array}{l}\mathrm{BCS}^{\mathrm{a}} \\
\text { mastectomy }^{\mathrm{b}}\end{array}$ & $\begin{array}{l}12(38.7 \%) \\
18(58 \%)\end{array}$ & $\begin{array}{l}14(45.2 \%) \\
14(45.2 \%)\end{array}$ & \multirow[t]{3}{*}{$\left(\mathrm{BCS}^{\mathrm{a}}\right.$ vs other) $0.448^{\mathrm{m}}$} \\
\hline & & bilat mastectomy $^{\mathrm{c}}$ & $1(3.2 \%)$ & $1(3.2 \%)$ & \\
\hline & & subcut mastect ${ }^{\mathrm{d}}$ & $0(0 \%)$ & $2(6.5 \%)$ & \\
\hline & \multirow[t]{2}{*}{ Axilla } & $\begin{array}{l}x^{e} \\
S N L B^{f}\end{array}$ & $\begin{array}{l}1(3.2 \%) \\
4(12.9 \%)\end{array}$ & $1(3.2 \%)$ & \multirow[t]{2}{*}{$1^{\mathrm{n}}$} \\
\hline & & $\mathrm{ALND}^{\mathrm{g}}$ & $26(83.9 \%)$ & $27(87.1 \%)$ & \\
\hline \multirow[t]{12}{*}{ Oncological Treatment } & \multirow[t]{2}{*}{ Neoadj $^{\text {h }}$} & all & $15(48.4 \%)$ & $10(32.3 \%)$ & $0.196^{\mathrm{m}}$ \\
\hline & & followed by chemotx & $11(35.5 \%)$ & $6(19.4 \%)$ & $0.342^{\mathrm{m}}$ \\
\hline & \multirow[t]{2}{*}{ Chemotx $^{\mathrm{i}}$} & $\begin{array}{l}\text { yes } \\
\text { no }\end{array}$ & $\begin{array}{l}24(77.4 \%) \\
5(16.1 \%)\end{array}$ & $\begin{array}{l}24(77.4 \%) \\
7(22.6 \%)\end{array}$ & \multirow[t]{2}{*}{$0.605^{\mathrm{m}}$} \\
\hline & & $\mathrm{nd}^{\mathrm{o}}$ & $2(6.5 \%)$ & $0(0 \%)$ & \\
\hline & \multirow[t]{4}{*}{ Regimen $^{\mathrm{j}}$} & $\begin{array}{l}\text { antracyclin } \\
\text { antracyclin }+ \text { taxane }\end{array}$ & $\begin{array}{l}6(19.4 \%) \\
9(29 \%)\end{array}$ & $\begin{array}{l}7(22.6 \%) \\
9(29 \%)\end{array}$ & \multirow[t]{4}{*}{$0.839^{\mathrm{m}}$} \\
\hline & & trastuzumab & $4(12.9 \%)$ & $8(25.8 \%)$ & \\
\hline & & other & $5(16.1 \%)$ & $6(19.4 \%)$ & \\
\hline & & $\mathrm{nd}^{\mathrm{o}}$ & $4(12.9 \%)$ & $0(0 \%)$ & \\
\hline & \multirow[t]{2}{*}{ Radiotx $^{\mathrm{k}}$} & $\begin{array}{l}\text { yes } \\
\text { no }\end{array}$ & $\begin{array}{l}22(70.9 \%) \\
7(22.5 \%)\end{array}$ & $\begin{array}{l}22(70.9 \%) \\
9(29 \%)\end{array}$ & \multirow[t]{2}{*}{$0.668^{\mathrm{m}}$} \\
\hline & & $\mathrm{nd}^{\mathrm{o}}$ & $2(6.5 \%)$ & $0(0 \%)$ & \\
\hline & \multirow[t]{2}{*}{ Endocr ${ }^{1}$} & $\begin{array}{l}\text { yes } \\
\text { no }\end{array}$ & $\begin{array}{l}12(38.7 \%) \\
17(54.8 \%)\end{array}$ & $\begin{array}{l}24(77.4 \%) \\
7(22.6 \%)\end{array}$ & \multirow[t]{2}{*}{$0.004^{\mathrm{m}}$} \\
\hline & & $\mathrm{nd}^{\mathrm{o}}$ & $2(6.5 \%)$ & $0(0 \%)$ & \\
\hline
\end{tabular}

${ }^{\mathrm{a}}$ Breast conserving surgery, ${ }^{\mathrm{b}}$ Modified radical mastectomy, ${ }^{\mathrm{c}}$ Bilateral mastectomy, ${ }^{\mathrm{d}}$ Subcutaneous mastectomy, ${ }^{\mathrm{e}}$ No surgery, ${ }^{\mathrm{f}}$ Sentinel lymph node biopsy, ${ }^{\mathrm{g}}$ Axillary lymph node dissection, ${ }^{\mathrm{h}}$ Neoadjuvant chemotherapy, ${ }^{\mathrm{i}}$ Adjuvant chemotherapy, ${ }^{\mathrm{j}}$ Adjuvant chemotherapy regimen ${ }^{\mathrm{k}}$ Adjuvant radiotherapy, ${ }^{1}$ Adjuvant endocrine therapy, ${ }^{\mathrm{m}}$ Chi-square test, ${ }^{\mathrm{n}}$ Fisher's exact test, ${ }^{\mathrm{o}}$ No data 
Table 2 Relapse, survival and family history data of PABC and control patients
${ }^{\text {a }}$ No data,

${ }^{\mathrm{b}}$ Immunohistochemistry-based subtype, ${ }^{\mathrm{c}}$ Triple negative immunophenotype, ${ }^{\mathrm{d}}$ Breast cancer related family history, ${ }^{\mathrm{e}} \mathrm{Chi}-$ square test, ${ }^{\mathrm{f}}$ Fisher's exact test

\begin{tabular}{|c|c|c|c|c|c|}
\hline & & & $\begin{array}{l}\text { PABC } \\
N=31\end{array}$ & $\begin{array}{l}\text { Control } \\
N=31\end{array}$ & $p$-value \\
\hline \multirow[t]{7}{*}{ Relapse } & & yes & $18(58.1 \%)$ & $4(12.9 \%)$ & $0.0003^{\mathrm{f}}$ \\
\hline & & no & $12(38.7 \%)$ & $25(80.6 \%)$ & \\
\hline & & $\mathrm{nd}^{\mathrm{a}}$ & $1(3.2 \%)$ & $2(6.5 \%)$ & \\
\hline & Loco-regional & $\begin{array}{l}\text { yes } \\
\text { no }\end{array}$ & $\begin{array}{l}6(19.4 \%) \\
24(77.4 \%)\end{array}$ & $\begin{array}{l}1(3.2 \%) \\
28(90.3 \%)\end{array}$ & $0.102^{\mathrm{f}}$ \\
\hline & & $\mathrm{nd}^{\mathrm{a}}$ & $1(3.2 \%)$ & $2(6.5 \%)$ & \\
\hline & Systemic & $\begin{array}{l}\text { yes } \\
\text { no }\end{array}$ & $\begin{array}{l}14(45.2 \%) \\
16(51.6 \%)\end{array}$ & $\begin{array}{l}3(9.7 \%) \\
26(83.9 \%)\end{array}$ & $0.003^{\mathrm{f}}$ \\
\hline & & $\mathrm{nd}^{\mathrm{a}}$ & $1(3.2 \%)$ & $2(6.5 \%)$ & \\
\hline \multirow[t]{3}{*}{ Death } & Deceased & & $13(41.9 \%)$ & $2(6.5 \%)$ & \\
\hline & Alive & $\begin{array}{l}\text { All } \\
\text { alive with disease }\end{array}$ & $\begin{array}{l}18(58.1 \%) \\
1(3.2 \%)\end{array}$ & $\begin{array}{l}29(93.5 \%) \\
2(6.5 \%)\end{array}$ & $0.005^{\mathrm{e}}$ \\
\hline & & no evidence of disease & $17(54.8 \%)$ & $27(87.1 \%)$ & \\
\hline \multirow[t]{5}{*}{ Death/IHC subtype ${ }^{b}$} & & LumA & $0 / 0$ & $0 / 5$ & See \\
\hline & & LumBprol & $6 / 10$ & $0 / 8$ & Fig. 5 \\
\hline & & LumBHer2 & $1 / 3$ & $0 / 6$ & \\
\hline & & Her2+ & $1 / 3$ & $2 / 5$ & \\
\hline & & $\mathrm{TNBC}^{\mathrm{c}}$ & $5 / 15$ & $0 / 7$ & \\
\hline \multirow[t]{5}{*}{ Family history } & All & positive & $14(45.2 \%)$ & $15(48.4 \%)$ & $0.558^{\mathrm{e}}$ \\
\hline & & negative & $7(22.6 \%)$ & $5(16.1 \%)$ & \\
\hline & & $\mathrm{nd}^{\mathrm{a}}$ & $10(32.3 \%)$ & $11(35.5 \%)$ & \\
\hline & $\mathrm{BC}$ related ${ }^{\mathrm{d}}$ & $\begin{array}{l}\text { positive } \\
\text { negative }\end{array}$ & $\begin{array}{l}8(25.8 \%) \\
13(41.9 \%)\end{array}$ & $\begin{array}{l}7(22.6 \%) \\
13(41.9 \%)\end{array}$ & $0.837^{\mathrm{e}}$ \\
\hline & & $\mathrm{nd}^{\mathrm{a}}$ & $10(32.3 \%)$ & $11(35.5 \%)$ & \\
\hline
\end{tabular}

however administered twice as frequently to controls ( $p=$ 0.004).

\section{Relapse and Survival Comparison of PABC and Control Patients}

Table 2 summarizes relapse, survival and family history data of PABC and controls.

Relapse was significantly more common in the PABC group ( $p=0.003), 14$ (45.2\%) patients had systemic relapse as compared to three $(9.7 \%)$ control patients $(p=0.0003)$. Systemic relapse was the most common in LumBprol (6 patients out of 10) and triple negative cases (5 patients out of 15); all these patients died of their disease. Disease free and overall survival was significantly worse in PABC cases ( $p=$ 0.0004 and $p=0.0007$ ) (Fig. $1 \mathrm{a}$ and $\mathrm{c}$ ). When survival data of pregnant and postpartum patients were assessed separately, the postpartum patient group showed significantly worse disease free $(p=0.001)$ and overall survival $(p=0.00008)$ as compared with the controls. Disease free survival of pregnant patients was inferior in comparison to control cases ( $p=$ 0.007 ), but overall survival was not significantly worse (Fig. 1b and d). The outcome of PABC was inferior, since thirteen PABC patients $(41.9 \%$ ) died of the disease; 11 of whom had postpartum breast cancer, while 2 patients $(6.5 \%)$ died in the control group $(p=0.005)$ (Fig. 2). There was no difference between the PABC and non-PABC group when overall and breast cancer related family history was evaluated.

\section{Pathological Data Analysis}

Table 3 summarizes the tumor characteristics of PABC and control patients.

The most common tumor type was high grade invasive ductal carcinoma (IDC) in both groups. There was no significant difference in tumor $\mathrm{T}$ or $\mathrm{N}$ stage. For assessment of median size of the invasive tumor, when patients were treated with neoadjuvant chemotherapy, tumor size before treatment was considered. Median size of PABC was $24 \mathrm{~mm}$ (range 10-100 $\mathrm{mm}$ ) compared to that of controls (22 $\mathrm{mm}$; range 9-85 $\mathrm{mm}, p=0.13$ ) (Supplementary Material 2). The Notthingham Prognostic Index (NPI) median value for PABC was 6 (range: 3.24 8.5), and 4.65 (range: 3.24-7.2) for controls (Fig. 3). This finding categorized PABC as a disease of poor prognosis, while the non-PABC group was categorized as having intermediate prognosis $(p=0.03)$. Patients who had complete or partial pathological response after neoadjuvant chemotherapy were excluded from this analysis. 

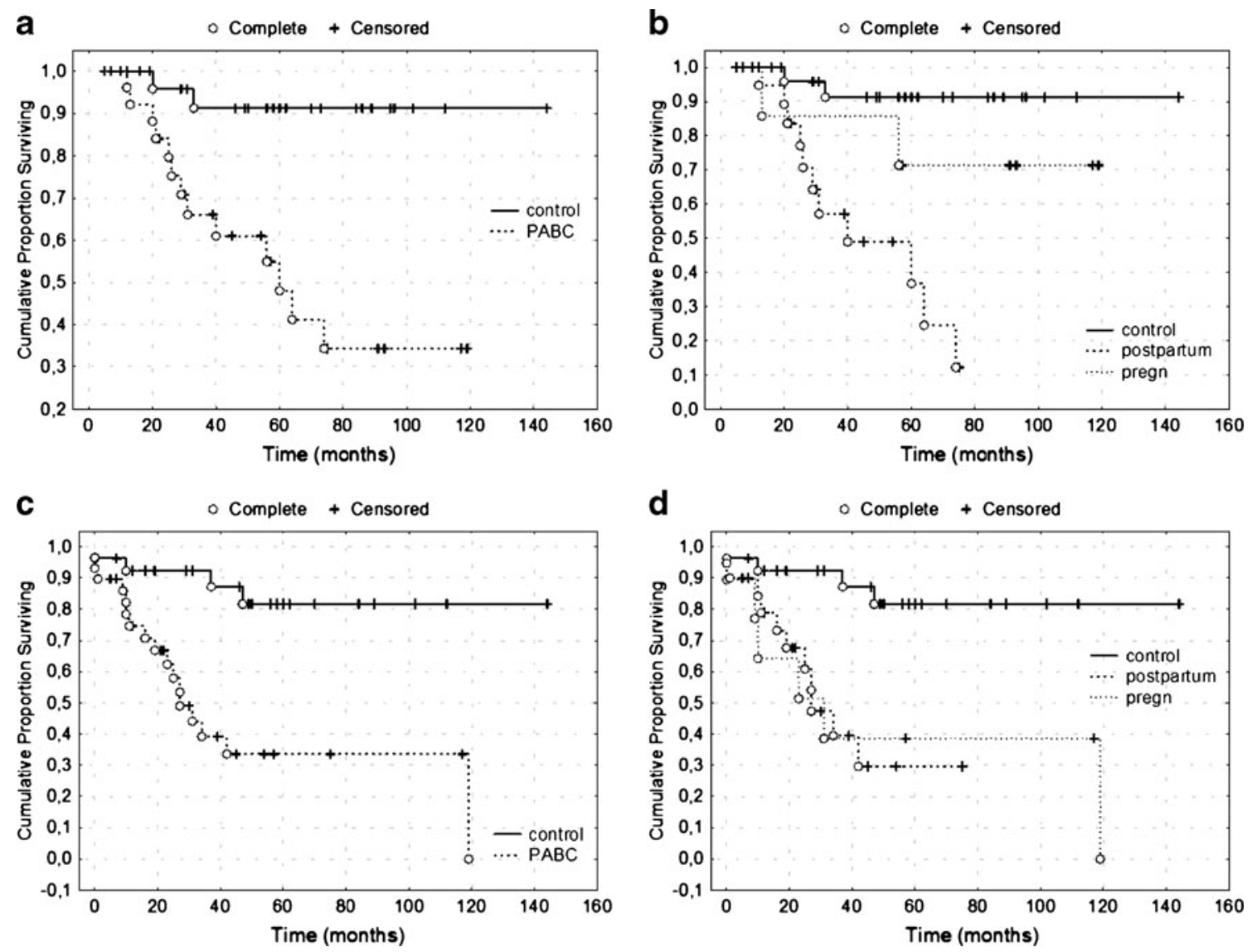

Fig. 1 a Overall survival of PABC and control patients $(p=0.0007)$. b Overall survival of pregnant, postpartum and control patients $(p=0.002)$ (control vs. postpartum $p=0.00008$, control vs. pregnant $p=0.203$, postpartum vs. pregnant $p=0.049$ ). $\mathbf{c}$ Disease free survival of PABC and

Lympho-vascular invasion was detected in $61.3 \%$ of cases and $48.4 \%$ of controls $(p=0.228)$.

PABC and control cases were ER negative in $58 \%$ and $38.8 \%$, respectively. The difference in PgR expression was significant: $87.1 \%$ of PABC cases, whereas $61.3 \%$ of controls did not express PgR $(p=0.04)$. No significant difference was noticed in Her2 status and by p53 immunostaining. All PABC

control patients $(p=0.0004)$. $\mathbf{d}$ Disease free survival of pregnant, postpartum and control patients $(p=0.007)$ (control vs. postpartum $p=0.001$, control vs. pregnant $p=0.007$, postpartum vs. pregnant $p=0.979$ )

cases were highly proliferating by Ki67 labeling index $(p=0.01)$. When analyzing IHC-based subtypes, triple negative tumors predominated in PABC (48.4 \%), followed by LumBprol tumors $(32.3 \%)$. There were no LumA tumors in this group.

Associated in situ lesions showed significant differences in patient cases and controls $(p=0.017)$. In $45.2 \%$ of PABC cases, the invasive tumor was associated with high grade

Fig. 2 a IHC-based subtype and death of PABC and control patients (n: number of patients). $\mathbf{b}$ IHC-based subtype and death of pregnant, postpartum and control patients (n: number of patients) a

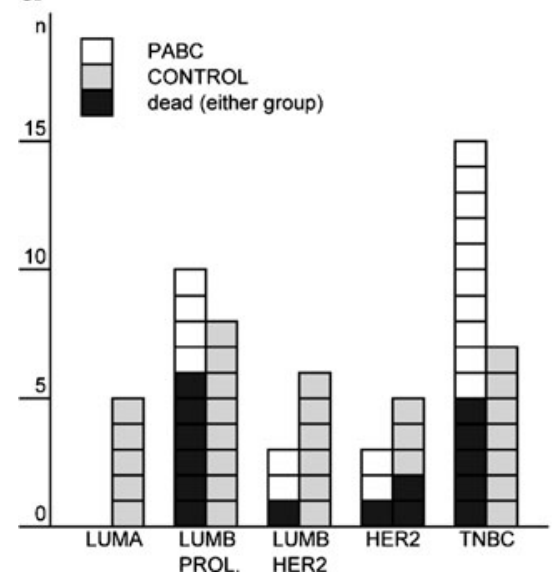

b

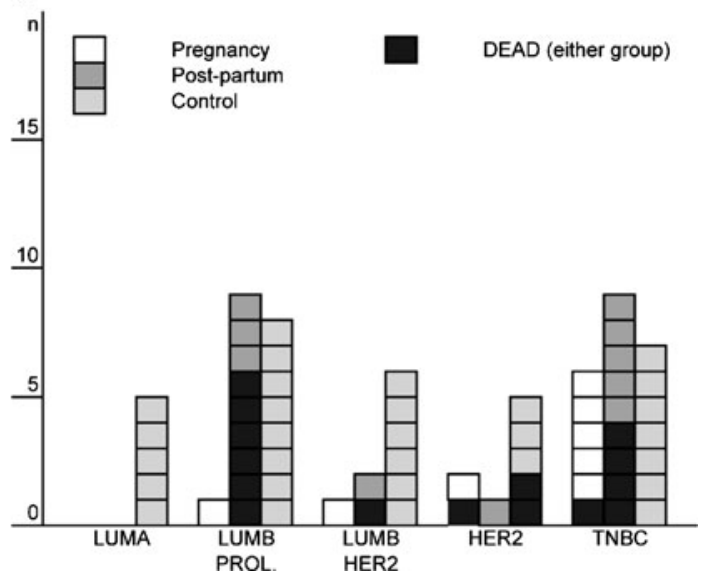


Table 3 Tumor characteristics of PABC and control cases

\begin{tabular}{|c|c|c|c|c|}
\hline & & $\begin{array}{l}\text { PABC } \\
N=31\end{array}$ & $\begin{array}{l}\text { Control } \\
N=31\end{array}$ & $p$-value \\
\hline Type & $\begin{array}{l}\text { IDC }^{\mathrm{a}} \\
\text { Other }\end{array}$ & $\begin{array}{l}26(83.9 \%) \\
5(16.1 \%)\end{array}$ & $\begin{array}{l}30(96.8 \%) \\
1(3.2 \%)\end{array}$ & $0.195^{\mathrm{p}}$ \\
\hline \multirow[t]{4}{*}{ Associated in situ carcinoma } & $\begin{array}{l}\text { none } \\
\text { EIC non-high }^{\text {b }}\end{array}$ & $\begin{array}{l}11(35.5 \%) \\
3(9.7 \%)\end{array}$ & $\begin{array}{l}14(45.2 \%) \\
3(9.7 \%)\end{array}$ & \multirow[t]{4}{*}{$0.017^{\circ}$} \\
\hline & $\mathrm{EIC} \mathrm{high}^{\mathrm{c}}$ & $14(45.2 \%)$ & $3(9.7 \%)$ & \\
\hline & DCIS non-high $^{\mathrm{d}}$ & $1(3.2 \%)$ & $4(12.9 \%)$ & \\
\hline & DCIS high $^{\mathrm{e}}$ & $2(6.5 \%)$ & $7(22.6 \%)$ & \\
\hline Multifocal disease & $\begin{array}{l}\text { No } \\
\text { Yes }\end{array}$ & $\begin{array}{l}24(77.4 \%) \\
7(22.6 \%)\end{array}$ & $\begin{array}{l}28(90.3 \%) \\
3(9.7 \%)\end{array}$ & $0.306^{\mathrm{p}}$ \\
\hline \multirow[t]{2}{*}{ Grade } & $\begin{array}{l}1 \\
2\end{array}$ & $\begin{array}{l}0(0 \%) \\
5(16.1 \%)\end{array}$ & $\begin{array}{l}2(6.5 \%) \\
8(25.8 \%)\end{array}$ & \multirow[t]{2}{*}{$0.199^{\circ}$} \\
\hline & 3 & $26(83.9 \%)$ & $21(67.7 \%)$ & \\
\hline \multirow[t]{6}{*}{$\mathrm{T}$} & $\begin{array}{l}\mathrm{T} 1 \\
\mathrm{~T} 2\end{array}$ & $\begin{array}{l}6(19.4 \%) \\
9(29 \%)\end{array}$ & $\begin{array}{l}8(25.8 \%) \\
12(38.7 \%)\end{array}$ & \multirow[t]{6}{*}{$0.522^{\circ}$} \\
\hline & $\mathrm{T} 3$ & $1(3.2 \%)$ & $1(3.2 \%)$ & \\
\hline & yT0 & $2(6.5 \%)$ & $3(9.7 \%)$ & \\
\hline & yT1 & $5(16.3 \%)$ & $4(12.9 \%)$ & \\
\hline & yT2 & $4(12.9 \%)$ & $3(9.7 \%)$ & \\
\hline & yT3 & $4(12.9 \%)$ & $0(0 \%)$ & \\
\hline \multirow[t]{8}{*}{$\mathrm{N}$} & $\begin{array}{l}\mathrm{Nx} \\
\mathrm{N} 0\end{array}$ & $\begin{array}{l}1(3.2 \%) \\
6(19.4 \%)\end{array}$ & $\begin{array}{l}1(3.2 \%) \\
9(29 \%)\end{array}$ & \multirow[t]{8}{*}{$0.646^{\mathrm{o}}$} \\
\hline & N1 & $4(12.9 \%)$ & $7(22.6 \%)$ & \\
\hline & $\mathrm{N} 2$ & $4(12.9 \%)$ & $2(6.5 \%)$ & \\
\hline & $\mathrm{N} 3$ & $2(6.5 \%)$ & $2(6.5 \%)$ & \\
\hline & yN0 & $4(12.9 \%)$ & $5(16.1 \%)$ & \\
\hline & yN1 & $6(19.4 \%)$ & $4(12.9 \%)$ & \\
\hline & $\mathrm{yN} 2$ & $2(6.5 \%)$ & $1(3.2 \%)$ & \\
\hline & $\mathrm{yN} 3$ & $2(6.5 \%)$ & $0(0 \%)$ & \\
\hline ER & $\begin{array}{l}\text { ER+ } \\
\text { ER- }\end{array}$ & $\begin{array}{l}13(41.9 \%) \\
18(58.1 \%)\end{array}$ & $\begin{array}{l}19(61.3 \%) \\
12(38.7 \%)\end{array}$ & $0.127^{\circ}$ \\
\hline PgR & $\begin{array}{l}\text { PgR+ } \\
\text { PgR- }\end{array}$ & $\begin{array}{l}4(12.9 \%) \\
27(87.1 \%)\end{array}$ & $\begin{array}{l}12(38.7 \%) \\
19(61.3 \%)\end{array}$ & $0.04^{\mathrm{p}}$ \\
\hline \multicolumn{2}{|l|}{$\begin{array}{l}E R+P g R+ \\
E R+\text { PgR- }\end{array}$} & $\begin{array}{l}4 \\
9\end{array}$ & $\begin{array}{l}12 \\
7\end{array}$ & $0.149^{p}$ \\
\hline Her2 & $\begin{array}{l}\text { Her2+ } \\
\text { Her2- }\end{array}$ & $\begin{array}{l}6(19.4 \%) \\
25(80.6 \%)\end{array}$ & $\begin{array}{l}11(35.5 \%) \\
20(64.5 \%)\end{array}$ & $0.155^{\circ}$ \\
\hline \multirow[t]{2}{*}{ Ki67 } & $\begin{array}{l}\text { high } \\
\text { low }\end{array}$ & $\begin{array}{l}31(100 \%) \\
0(0 \%)\end{array}$ & $\begin{array}{l}24(77.4 \%) \\
6(19.4 \%)\end{array}$ & \multirow[t]{2}{*}{$0.01^{\mathrm{p}}$} \\
\hline & $\mathrm{nd}^{\mathrm{r}}$ & $0(0 \%)$ & $1(3.2 \%)$ & \\
\hline \multirow[t]{2}{*}{ P53 } & $\begin{array}{l}\text { positive } \\
\text { negative }\end{array}$ & $\begin{array}{l}15(48.4 \%) \\
9(29.3 \%)\end{array}$ & $\begin{array}{l}17(54.8 \%) \\
12(38.8 \%)\end{array}$ & \multirow[t]{2}{*}{$0.774^{\circ}$} \\
\hline & $n d^{r}$ & $7(22.6 \%)$ & $2(6.5 \%)$ & \\
\hline \multirow[t]{5}{*}{$\mathrm{NPI}^{\mathrm{f}}$} & median (range) & $6(3.24-8.5)$ & $4.65(3.24-7.2)$ & $0.03^{\mathrm{q}}$ \\
\hline & $\begin{array}{l}\text { excellent }(2-2.4)^{\mathrm{g}} \\
\operatorname{good}(>2.4-3.4)^{\mathrm{h}}\end{array}$ & $\begin{array}{l}0(0 \%) \\
2(6.5 \%)\end{array}$ & $\begin{array}{l}0(0 \%) \\
5(16.1 \%)\end{array}$ & \multirow[t]{4}{*}{$0.172^{\circ}$} \\
\hline & intermed $(>3.4-5.4)^{\mathrm{i}}$ & $7(22.6 \%)$ & $12(38.8 \%)$ & \\
\hline & $\operatorname{poor}(>5.4)^{\mathrm{j}}$ & $14(45.1 \%)$ & $9(29 \%)$ & \\
\hline & $n a^{\mathrm{k}}$ & $8(25.8 \%)$ & $5(16.1 \%)$ & \\
\hline \multirow[t]{2}{*}{$\mathrm{LVI}^{1}$} & no & $10(32.3 \%)$ & $15(48.4 \%)$ & \multirow[b]{2}{*}{$0.228^{\circ}$} \\
\hline & $\begin{array}{l}\text { yes } \\
\text { nd }^{\text {r }}\end{array}$ & $\begin{array}{l}19(61.3 \%) \\
2(6.5 \%)\end{array}$ & $\begin{array}{l}15(48.4 \%) \\
1(3.2 \%)\end{array}$ & \\
\hline
\end{tabular}


Table 3 (continued)

\begin{tabular}{|c|c|c|c|c|}
\hline & & $\begin{array}{l}\text { PABC } \\
N=31\end{array}$ & $\begin{array}{l}\text { Control } \\
N=31\end{array}$ & $p$-value \\
\hline \multirow[t]{5}{*}{ IHC-Subtype $^{\mathrm{m}}$} & LumA & $0(0 \%)$ & $5(16.1 \%)$ & \multirow[t]{5}{*}{$0.015^{\mathrm{o}}$} \\
\hline & LumBprol & $10(32.3 \%)$ & $8(25.8 \%)$ & \\
\hline & LumBHer2 & $3(9.7 \%)$ & $6(19.4 \%)$ & \\
\hline & Her2 & $3(9.7 \%)$ & $5(16.1 \%)$ & \\
\hline & $\mathrm{TNBC}^{\mathrm{n}}$ & $15(48.4 \%)$ & $7(22.6 \%)$ & \\
\hline
\end{tabular}

${ }^{\mathrm{a}}$ Invasisve ductal carcinoma, ${ }^{\mathrm{b}}$ Extensive intraductal carcinoma component non-high nuclear grade, ${ }^{\mathrm{c}}$ Extensive intraductal carcinoma component high nuclear grade, ${ }^{\mathrm{d}}$ Ductal carcinoma in situ non-high nuclear grade, ${ }^{\mathrm{e}}$ Ductal carcinoma in situ high nuclear grade, ${ }^{\mathrm{f}}$ Nottingham Prognostic Index, ${ }^{\mathrm{g}}$ Excellent prognosis by NPI, ${ }^{\mathrm{h}}$ Good prognosis by NPI, ${ }^{\mathrm{i}}$ Intermediate prognosis by NPI, ${ }^{\mathrm{j}}$ Poor prognosis by NPI, ${ }^{\mathrm{k}}$ NPI not assessable- partial or complete pathological response after neoadjuvant chemotherapy, ${ }^{1}$ Lympho-vascular invasion, ${ }^{\mathrm{m}}$ Immunohistochemistry-based subtype, ${ }^{\mathrm{n}}$ Triple negative immunophenotype, ${ }^{\mathrm{o}}$ Chi-square test, ${ }^{\mathrm{p}}$ Fisher's exact test, ${ }^{\mathrm{q}}$ Wilcoxon test, ${ }^{\mathrm{r}}$ No data

extensive intraductal carcinoma component as compared with $9.7 \%$ in controls. Forty-five percent of controls did not have in situ carcinoma associated with the invasive tumor. The predominant in situ lesion of controls was focal, high grade ductal carcinoma in situ $(22.6 \%)$.

Upon multivariate Cox proportional model analysis pregnancy related status was associated with both relapse and survival, while age was associated with relapse (Supplementary Material 3).

\section{Discussion}

As far as outcome of PABC is concerned data are conflicting: some authors showed no difference in outcome of PABC and non-PABC cases [14, 16, 24], while others consider PABC as having unfavorable prognosis [1, 7-9]. In a recent metaanalysis of 30 studies, Azim et al. found that PABC patients had poor overall survival especially if breast cancer was diagnosed within 1 year postpartum [25]. Our study also

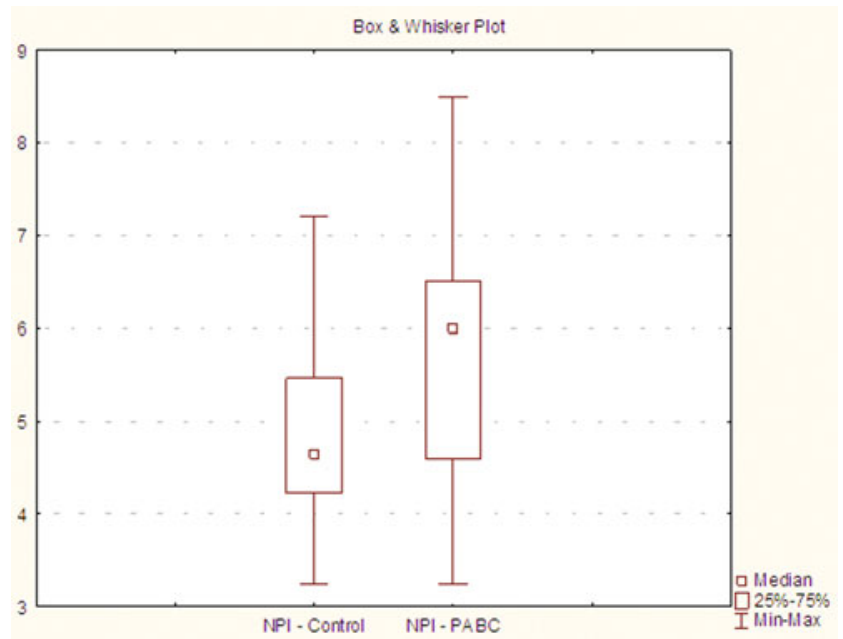

Fig. 3 Nottingham Prognostic Index of PABC and control cases $(p=0.03$, Wilcoxon $)$ demonstrates inferior outcome of PABC patients, systemic relapse and death being significantly more common in this group. Disease free survival of both pregnant and postpartum patients was worse than that of controls, and overall survival of the postpartum group was inferior compared to that of controls.

Previous studies have addressed the hypothesis that the unfavorable outcome of PABC is related to delayed diagnosis resulting in advanced disease at presentation $[2,26]$. We found no significant delay in surgery and initiation of oncological treatment of PABC versus control patients, although we could not assess the time elapsing between manifestation of first symptoms and time of initial diagnosis. Disease at presentation (size, T, N) was not found to be significantly different between the two groups; treatment modalities (surgery, neoadjuvant or adjuvant treatment frequency or regimen) did not differ except for the frequency of endocrine therapy, which was more common in the control group.

NPI is a continuous variable that combines several prognostic factors of BC (grade, tumor size, number of metastatic lymph nodes) [27, 28]. Although there was no significant difference between PABC and non-PABC when tumor size, $\mathrm{T}, \mathrm{N}$ and grade at initial presentation were assessed separately, the combination of these factors by NPI resulted in significant difference: based on median NPI value the PABC group was categorized as having poor prognosis, while the non-PABC group as having intermediate prognosis. This finding suggests differences in tumor aggressiveness, which can not be captured by individual assessment of classical histopathological prognostic factors.

Progesterone receptor expression showed significant difference between PABC cases and controls, with PABC being mainly PgR negative. Although there were more ER + PgRLuminalB tumors in PABC patients, the difference was not statistically significant, thus the detected difference in $\mathrm{PgR}$ expression is driven mainly by the over-representation of triple negative tumors in PABC, since Her2 expression did not show major differences. 
Ki67 labeling index was above $15 \%$ in all PABC cases, reflected also by the difference in IHC-based tumor subtypes, namely that there were no LumA tumors in this group. From all PABC cases $48.4 \%$ showed triple negative and $32.3 \%$ LumBprol phenotype. These two subtypes represented around $80 \%$ of all PABC cases, while only $48 \%$ of the control cases. Our finding on the over-representation of TNBC in PABC is in concordance with that of other authors [18]. Survival assessed by IHC-based subtypes showed the worst survival of patients with LumBprol and triple negative phenotype, especially in postpartum cases. These findings underline the importance of Ki67 testing in ER + Her2- tumors, in order to detect tumors of LumBprol subtype with an eventual worse prognosis.

Associated ductal in situ lesions are known to have an impact on local control of breast cancer. There is a higher risk of local recurrence if ductal carcinoma in situ demonstrates high nuclear grade, comedo necrosis, is extensive, involves surgical margins or the patient is young [29-31]. These factors are not entirely unrelated though, since young patients ( $\leq 45$ years) tend to have high nuclear grade in situ lesions with comedo necrosis and the extent of DCIS is significantly greater compared to older women [29, 31].

In our study, PABC as compared with non-PABC was more often associated with an extensive intraductal carcinoma component showing high nuclear grade and comedo necrosis, but there was no difference in local relapse. Our finding on the one hand can underline the unfavorable biology of PABC, since increasing molecular evidence suggest that high grade in situ ductal lesions harbour complex and heterogeneous genomic alterations that are highly similar to that of high grade IDC and these aberrations are different from that of low grade DCIS and IDC $[32,33]$. On the other hand, this finding can also incite thoughts about the significantly more extensive involvement of ducts by high grade in situ lesions in PABC, which might be a further consequence of the altered milieu related to pregnancy and the postpartum condition.

Possible explanations for the detected outcome differences between PABC and non-PABC besides different tumor biology may involve the relative immunosuppressed state associated with pregnancy, the altered hormonal milieu (the placenta and the fetus as new endocrine "organs", higher estrogen, progesterone and growth hormone levels during pregnancy, increased prolactin level during lactation). Changes in tumor microenvironment during pregnancy (increased responsiveness of the breast to growth factor stimulation) or during postpartum remodeling - when extracellular matrix modifications associated with post-lactational involution create a milieu that resembles wound-healing [34] - are all known factors enhancing tumor growth and metastasis. O'Brien et al. have demonstrated the role of post-lactational involutionassociated, alternatively activated M2-type macrophages that share characteristics with Tumor-associated macrophages
(TAMs). TAMs, by secreting growth factors, enhance tumor growth. Immature macrophages might also suppress cytotoxic T-cell function, thus creating an immunosuppressed microenvironment $[35,36]$. Fornetti et al. have proposed a model showing how post-lactational epithelial cells transition to become phagocytic and engulf the apoptotic (formerly milkproducing) cells during involution while secreting cytokines and growth factors, thus resulting in a tumor-supportive microenvironment [34].

The weakness of our study is its retrospective nature and the lack of information on the time elapsed between first symptoms and first diagnosis of breast cancer. Its strength is the combination of management data and survival analysis with detailed histopathology and IHC data analysis, including not only HR status, but Her2, Ki67, p53, IHC-based subtype, NPI and associated in situ lesion analysis as well. To the best of our knowledge these factors have not been investigated so far together in a matched case control study on PABC.

In conclusion, our study has demonstrated inferior outcome of PABC, especially when detected postpartum. Unfavorable tumor biology was reflected by the predominance of triple negative and LumBprol tumors and PABC was commonly associated with high grade EIC. To our best knowledge, this latter finding has not been previously addressed. Individual analysis of classical prognostic factors could not detect inferior prognosis of $\mathrm{PABC}$, but combination of these factors by NPI has predicted the aggressive nature and poor prognosis of pregnancy associated breast cancer. Based on the literary data, it seems that besides tumor biology, the tumor microenvironment altered by pregnancy and the postpartum state, most probably also plays important role in the inferior outcome of the disease. This issue is still underinvestigated though, new treatments (e.g. successful targeting of tumor microenvironment) however might contribute to improving the prognosis of PABC.

Acknowledgments The authors are grateful to Gábor Lotz MD $\mathrm{PhD}$ for supervising HER2 FISH assessment.

Conflict of interest The authors declare that they have no conflicts of interest.

\section{References}

1. Johansson ALV, Andersson TM-L, Hsieh C-C, Cnattingius S, Lambe $M$ (2011) Increased mortality in women with breast cancer detected during pregnancy and different periods postpartum. Cancer Epidemiol Biomark Prev 20(9):1865-1872. doi:10.1158/10559965.epi-11-0515

2. Woo Jc YTHTC (2003) Breast cancer in pregnancy: a literature review. Arch Surg 138(1):91-98. doi:10.1001/archsurg.138.1.91 
3. Lyons T, Schedin P, Borges V (2009) Pregnancy and breast cancer: when they collide. J Mammary Gland Biol Neoplasia 14(2):87-98. doi:10.1007/s10911-009-9119-7

4. Liu Q, Wuu J, Lambe M, Hsieh S-F, Ekbom A, Hsieh C-C (2002) Transient increase in breast cancer risk after giving birth: postpartum period with the highest risk (Sweden). Cancer Causes Control 13(4): 299-305. doi:10.1023/a:1015287208222

5. Lambe M, Hsieh C, Trichopoulos D, Ekbom A, Pavia M, Adami HO (1994) Transient increase in the risk of breast cancer after giving birth. New England J Med 331(1):5-9. doi:10.1056/NEJM19940 7073310102

6. Nugent P, Connell TXO (1985) Breast cancer and pregnancy. Arch Surg 120(11):1221-1224

7. Ali SA, Gupta S, Sehgal R, Vogel V (2012) Survival outcomes in pregnancy associated breast cancer: a retrospective case control study. Breast J 18(2):139-144. doi:10.1111/j.1524-4741.2011. 01201.x

8. Moreira WB, Brandão EC, Soares AN, Lucena CEM, Antunes CMF (2010) Prognosis for patients diagnosed with pregnancy-associated breast cancer: a paired case-control study. Sao Paulo Med J 128:119 124

9. Bonnier P, Romain S, Dilhuydy JM, Bonichon F, Julien JP, Charpin C, Lejeune C, Martin PM, Piana L (1997) Influence of pregnancy on the outcome of breast cancer: a case-control study. Int J Cancer 72(5):720-727. doi:10.1002/(sici)1097-0215(19970904)72:5<720:: aid-ijc3 $>3.0 . c 0 ; 2-u$

10. Azim HA, Santoro L, Russell-Edu W, Pentheroudakis G, Pavlidis N, Peccatori FA (2012) Prognosis of pregnancyassociated breast cancer: a meta-analysis of 30 studies. Cancer Treat Rev 38(7):834-842

11. Anders CK, Hsu DS, Broadwater G, Acharya CR, Foekens JA, Zhang Y, Wang Y, Marcom PK, Marks JR, Febbo PG, Nevins JR, Potti A, Blackwell KL (2008) Young age at diagnosis correlates with worse prognosis and defines a subset of breast cancers with shared patterns of gene expression. J Clin Oncol 26(20):3324-3330. doi:10. 1200/jco.2007.14.2471

12. Middleton LP, Amin M, Gwyn K, Theriault R, Sahin A (2003) Breast carcinoma in pregnant women. Cancer 98(5):1055-1060. doi:10. 1002/cncr.11614

13. Hahn KME, Johnson PH, Gordon N, Kuerer H, Middleton L, Ramirez M, Yang W, Perkins G, Hortobagyi GN, Theriault RL (2006) Treatment of pregnant breast cancer patients and outcomes of children exposed to chemotherapy in utero. Cancer 107(6):12191226. doi:10.1002/cncr.22081

14. Halaska MJ, Pentheroudakis G, Strnad P, Stankusova H, Chod J, Robova H, Petruzelka L, Rob L, Pavlidis N (2009) Presentation, management and outcome of 32 patients with pregnancy-associated breast cancer: a matched controlled study. Breast J 15(5):461-467. doi:10.1111/j.1524-4741.2009.00760.x

15. Azim HA Jr, Botteri E, Renne G, Dell'Orto P, Rotmensz N, Gentilini O, Sangalli C, Pruneri G, Di Nubila B, Locatelli M, Sotiriou C, Piccart M, Goldhirsch A, Viale G, Peccatori FA (2012) The biological features and prognosis of breast cancer diagnosed during pregnancy: a case-control study. Acta Oncol 51(5):653-661. doi:10. 3109/0284186X.2011.636069

16. Murphy CG, Mallam D, Stein S, Patil S, Howard J, Sklarin N, Hudis CA, Gemignani ML, Seidman AD (2012) Current or recent pregnancy is associated with adverse pathologic features but not impaired survival in early breast cancer. Cancer 118(13):3254-3259. doi:10. 1002/cncr.26654

17. Genin A-S, Lesieur B, Gligorov J, Antoine M, Selleret L, Rouzier R (2012) Pregnancy-associated breast cancers: do they differ from other breast cancers in young women? Breast Edinb Scotl 21(4):550-555

18. Pilewskie M, Gorodinsky P, Fought A, Hansen N, Bethke K, Jeruss J, Scholtens D, Khan S (2012) Association between recency of last pregnancy and biologic subtype of breast cancer. Ann Surg Oncol 19(4):1167-1173. doi:10.1245/s10434011-2104-6

19. Reed W, Hannisdal E, Skovlund E, Thoresen S, Lilleng P, Nesland JM (2003) Pregnancy and breast cancer: a population-based study. Virchows Archiv 443(1):44-50. doi:10.1007/s00428-003-0817-z

20. Hammond MEH, Hayes DF, Dowsett M, Allred DC, Hagerty KL, Badve S, Fitzgibbons PL, Francis G, Goldstein NS, Hayes M, Hicks DG, Lester S, Love R, Mangu PB, McShane L, Miller K, Osborne CK, Paik S, Perlmutter J, Rhodes A, Sasano H, Schwartz JN, Sweep FCG, Taube S, Torlakovic EE, Valenstein P, Viale G, Visscher D, Wheeler T, Williams RB, Wittliff JL, Wolff AC (2010) American Society of Clinical Oncology/College of American Pathologists Guideline Recommendations for Immunohistochemical Testing of Estrogen and Progesterone Receptors in breast cancer. J Clin Oncol 28(16):2784-2795. doi:10.1200/jco. 2009.25.6529

21. Wolff AC, Hammond MEH, Schwartz JN, Hagerty KL, Allred DC, Cote RJ, Dowsett M, Fitzgibbons PL, Hanna WM, Langer A, McShane LM, Paik S, Pegram MD, Perez EA, Press MF, Rhodes A, Sturgeon C, Taube SE, Tubbs R, Vance GH, van de Vijver M, Wheeler TM, Hayes DF (2007) American Society of Clinical Oncology/College of American Pathologists Guideline Recommendations for Human Epidermal Growth Factor Receptor 2 testing in breast cancer. Arch Pathol Lab Med 131(1):18-43. doi:10. 1043/1543-2165(2007)131[18:asocco]2.0.co;2

22. Cheang MCU, Chia SK, Voduc D, Gao D, Leung S, Snider J, Watson M, Davies S, Bernard PS, Parker JS, Perou CM, Ellis MJ, Nielsen TO (2009) Ki67 Index, HER2 status, and prognosis of patients with luminal B breast cancer. J Nat Cancer Inst 101(10):736-750. doi: 10.1093/jnci/djp082

23. Goldhirsch A, Wood WC, Coates AS, Gelber RD, Thürlimann B, Senn H-J, Members P (2011) Strategies for subtypes-dealing with the diversity of breast cancer: highlights of the St Gallen International Expert Consensus on the primary therapy of early breast cancer 2011. Ann Oncol 22(8):1736-1747. doi:10.1093/ annonc/mdr304

24. Beadle BM, Woodward WA, Middleton LP, Tereffe W, Strom EA, Litton JK, Meric-Bernstam F, Theriault RL, Buchholz TA, Perkins GH (2009) The impact of pregnancy on breast cancer outcomes in women $\leq 35$ years. Cancer 115(6):1174-1184. doi:10.1002/cncr.24165

25. Azim HA Jr, Santoro L, Russell-Edu W, Pentheroudakis G, Pavlidis N, Peccatori FA (2012) Prognosis of pregnancy-associated breast cancer: a meta-analysis of 30 studies. Cancer Treat Rev 38(7):834 842. doi:10.1016/j.ctrv.2012.06.004

26. Guinee F, Hess KR, Taylor SH, Olsson H, Möller T, Fahey T, Gladikov JV, van den Blink JW, Bonichon F, Dische S, Yates JW, Cleton $F$ (1994) Effect of pregnancy on prognosis for young women with breast cancer. Lancet 343(8913):1587-1589

27. Kollias J, Murphy C, Elston C, Ellis I, Robertson J, Blamey R (1999) The prognosis of small primary breast cancers. Eur J Cancer 35(6): 908-912

28. Albergaria A, Ricardo S, Milanezi F, Carneiro V, Amendoeira I, Vieira D, Cameselle-Teijeiro J, Schmitt F (2011) Nottingham prognostic index in triple-negative breast cancer: a reliable prognostic tool? BMC Cancer 11(1):299

29. Collins LC, Achacoso N, Nekhlyudov L, Fletcher SW, Haque R, Quesenberry CPJ, Puligandla B, Alshak NS, Goldstein LC, Gown AM, Schnitt SJ, Habel LA (2009) Relationship Between Clinical and Pathologic Features of Ductal Carcinoma In Situ and Patient Age: An Analysis of 657 Patients. The American Journal of Surgical Pathology 33 (12):1802-1808

30. Schnitt SJ (2010) Local outcomes in ductal carcinoma in situ based on patient and tumor characteristics. JNCI Monographs 2010(41): 158-161. doi:10.1093/jncimonographs/lgq031 
31. Goldstein NS, Vicini FA, Kestin LL, Thomas M (2000) Differences in the pathologic features of ductal carcinoma in situ of the breast based on patient age. Cancer 88(11):25532560. doi:10.1002/1097-0142(20000601)88:11<2553::AIDCNCR18>3.0.CO;2-V

32. Vincent-Salomon A, Lucchesi C, Gruel N, Raynal V, Pierron G, Goudefroye R, Reyal F, Radvanyi F, Salmon R, Thiery J-P, SastreGarau X, Sigal-Zafrani B, Fourquet A, Delattre O (2008) Integrated genomic and transcriptomic analysis of ductal carcinoma in situ of the breast. Clin Cancer Res 14(7):1956-1965. doi:10.1158/10780432.ccr-07-1465

33. Lopez-Garcia MA, Geyer FC, Lacroix-Triki M, Marchió C, ReisFilho JS (2010) Breast cancer precursors revisited: molecular features and progression pathways. Histopathology 57(2):171-192. doi:10. 1111/j.1365-2559.2010.03568.x

34. Fornetti J, Martinson H, Borges V, Schedin P (2012) Emerging targets for the prevention of pregnancy-associated breast cancer. Cell Cycle 11(4):639-640

35. O'Brien J, Schedin P (2009) Macrophages in breast cancer: do involution macrophages account for the poor prognosis of pregnancy-associated breast cancer? J Mammary Gland Biol Neoplasia 14(2):145-157. doi:10.1007/s10911-009-9118-8

36. O’Brien J, Lyons T, Monks J, Lucia MS, Wilson RS, Hines L, Y-g M, Borges V, Schedin P (2010) Alternatively activated macrophages and collagen remodeling characterize the postpartum involuting mammary gland across species. Am J Pathol 176(3):1241-1255 